J. S. INSUASTY

\title{
Supervivencia en cáncer de próstata y factores relacionados
}

\section{Survival in prostate cancer and related factors}

Aunque el cáncer de próstata es la segunda causa de muerte por cáncer en los hombres después del cáncer de pulmón en los EE.UU., tiene una excelente supervivencia específica para la mayoría de los pacientes (1). Debido a la edad de presentación en adultos mayores, a su detección temprana, a la eficacia del tratamiento y a su curso clínico prolongado para la mayoría de los hombres, los sobrevivientes de cáncer de próstata tienen mayor riesgo de muerte por causas no cancerosas, antes que por su neoplasia de base (1-3).

Villegas y cols. presentan en este número la supervivencia a cinco y diez años en 404 pacientes, sometidos a un seguimiento global de 40 meses, haciendo una descripción de correlación entre factores pronósticos y los diferentes resultados de supervivencia (4). En la población de pacientes estudiados, encuentran $41 \%$ de ellos estadio T1c con una supervivencia a diez años de $94 \%$ para las edades entre 61 y 70 años $(\mathrm{p}=0.001)$; la supervivencia en pacientes con Gleasson $\leq 7$ fue de $83 \%$, sin diferencia significativa entre los scores de Gleasson $4+3$ o $3+4(p=0.9698)$, siendo estos resultados similares a los publicados en la literatura latinoamericana (5); condición ésta que nos lleva a especular acerca de una posible "condición biológica" compartida que podría determinar este comportamiento. En este orden de ideas, Villegas y Cols. (4), encuentran supervivencia según el nivel sérico de PSA $<4 \mathrm{ng} / \mathrm{mL}$ de $98 \%$ y para PSA $>20-99 \mathrm{ng} / \mathrm{mL}$ de $78 \%$ ( $\mathrm{p}=0.001$ ). Finalmente reportan supervivencia a cinco y diez años en etapa localizada de 92 y 79\%, respectivamente; y 52 y $30 \%$ para enfermedad metastásica a cinco y diez años, respectivamente. Como se deja ver en la publicación en mención, el tratamiento actual del cáncer de próstata y su supervivencia, es el resultado de una serie de factores pronósticos, y otras variables que contribuyen a la obtención de un resultado, como se describe a continuación:

La falta de especificidad para el cáncer de próstata ha dado lugar a una gran controversia sobre el papel del examen sérico de rutina del PSA (antígeno prostático específico). La controversia aumenta por el conocimiento de que no todos los cánceres detectados por exámenes de rutina requieren tratamiento. Pero llamativamente la mayoría de pacientes a quienes se les realiza una biopsia de próstata, es porque cursan con elevación de los niveles séricos de PSA. En este orden de ideas, el diagnóstico de cáncer de próstata requiere de la obtención de una biopsia, la cual se realiza guiada por ecografía transrectal y a su vez debe estar precedida por la medición del PSA sérico (1-3).

Para realizar la biopsia de próstata se debe tener en cuenta la edad del paciente, el volumen prostático, los hallazgos al tacto digital de la próstata por vía rectal y a la historia familiar. De tal manera que un valor de PSA sustancialmente elevado por encima del nivel normal para cierta edad, podría ser una indicación para realizar una biopsia. Se debe considerar como sospechoso un cambio desde valores previos $>0.35 \mathrm{ng} / \mathrm{mL} /$ año en los PSA $<4.0$ o $0.75 \mathrm{ng} / \mathrm{mL}$ en los PSA $>4.0$, igualmente un examen anormal de la próstata por tacto rectal que muestre asimetría, superficie nodular, o induración es indicación para realizar una biopsia, independientemente de los valores de PSA sérico (1).

Para realizar el tratamiento del cáncer localizado de próstata, se hace una estratificación que en forma simplificada corresponde a: muy bajo riesgo, en el cual la enfermedad es detectada por biopsia de próstata basado solamente en PSA, examen digital o por imágenes de próstata normal, Gleasson $\leq 6,<3$ cilindros positivos, biopsia con compromiso $\leq 50 \%, \mathrm{PSA}<10 \mathrm{ng} /$ $\mathrm{mL}$, y densidad de PSA $<0.15$; bajo riesgo, en el cual la enfermedad es T1-T2a y PSA $<10 \mathrm{ng} /$ $\mathrm{mL}$, y Gleasson 6; riesgo intermedio T2b-c o PSA $10-20 \mathrm{ng} / \mathrm{mL}, \mathrm{o}$, Gleasson 7; y alto riesgo, en el cual la enfermedad es T3-T4 o PSA $>20$ $\mathrm{ng} / \mathrm{mL}$ o Gleasson 8-10. El enfoque inicial para cáncer de próstata localizado de muy bajo riesgo, y con una expectativa de vida $<20$ años (determinada por nomogramas o utilizando las tablas de cobertura de seguridad social www. ssa.gov/OAST/STAS/table4c6.htm) (1), se recomienda dejar al paciente en vigilancia activa antes que someterlo a un tratamiento activo inmediato, aunque este enfoque genera mucha ansiedad, y puede terminar llevando al
Ver artículo: página 101

Dr. Jesús S. Insuasty E.: InternistaOncólogo Clínico. Profesor Departamento de Medicina Interna, Universidad Industrial de Santander -UIS Oncólogo Clínico, Hospital Universitario de Santander. Insuasty Oncología e Investigación SAS Bucaramanga (Colombia). e-mail: jesusinsuastyasco@hotmail.com 
paciente a buscar algún tipo de intervención (prostatectomía o radioterapia), aun sin tener enfermedad progresiva. Para pacientes con cáncer de próstata de bajo riesgo y una expectativa de vida mayor a 10 años el tratamiento definitivo incluye prostatectomía radical, braquiterapia o radioterapia externa, y en casos muy seleccionados vigilancia activa (1). La elección de cualquier tratamiento específico requiere tener en cuenta los riesgos y beneficios relacionados con cada enfoque terapéutico, considerando las preferencias individuales y las comorbilidades. Para pacientes con expectativa menor a diez años se sugiere dejar al paciente en vigilancia activa.Aunque el control de la enfermedad con las dos modalidades terapéuticas de prostatectomía radical y radioterapia son similares, sí existen diferencias importantes en los patrones de toxicidad. Por ejemplo, después de la radioterapia son más frecuentes los síntomas irritativos y obstructivos, principalmente con la braquiterapia. Con la prostatectomía radical es más frecuente la presentación de incontinencia, que puede mejorar gradualmente. Los síntomas intestinales de frecuencia y urgencia son mas comunes después de la radioterpia o braquiterapia. La disfunción eréctil es más frecuente inmediatamente después de la prostatectomía radical, aunque la cirugía con preservación bilateral del nervio disminuye, pero no evita esta posibilidad. La disfunción eréctil también se presenta después de la radioterpia externa o braquiterapia y la incidencia aumenta después del tratamiento (1). Al terminar cualquier modalidad de tratamiento, el paciente debe continuar un seguimiento estricto, puesto que las recaídas son susceptibles de tratamientos exitosos.

El tratamiento inicial para cáncer de próstata regionalmente localizado de riesgo intermedio alto o muy alto, con expectativa de vida mayor a diez años y sin comorbilidades serias se recomienda radioterapia (externa, braquiterapia o la combinación de las dos) o prostatectomía radical (2). La radioterapia puede ser combinada, con un curso corto de tratamiento con deprivación de andrógenos DA (análogos de liberadores de hormona luteinizante RHLH + antiandrógenos) por cuatro a seis meses. Para hombres con expectativa de vida menor a diez años se recomienda radioterapia. Para cáncer de próstata de alto riesgo o muy alto riesgo, se indica tratamiento con radioterapia o radioterapia más braquiterapia o prostatectomía radical (si no existe fijación del tumor primario)
(2), cuando se usa radioterapia se recomienda combinar con DA a largo plazo. En pacientes sometidos a cirugía la presencia de factores adversos en la pieza quirúrgica indicaría la necesidad de tratamiento con deprivación androgénica adicional (ante extensión extracapsular, compromiso de vesículas seminales, márgenes positivos, compromiso ganglionar) o elevación persistente del PSA después de la cirugía, la radioterapia posoperatoria también puede estar indicada. Se puede usar terapia de DA o monoterapia antiandrogénica en pacientes con comorbilidad significativa que impida un tratamiento definitivo.

En pacientes con enfermedad metastásica ósea o visceral, el tratamiento es netamente paliativo con el propósito de prolongar la supervivencia, mejorar el dolor, mejorar la movilidad y prevenir complicaciones, tales como las fracturas patológicas y la compresión epidural. Se deben mantener los niveles de castración con testosterona sérica $\leq 50 \mathrm{ng} / \mathrm{dL}$, instaurar terapia antireabsortiva con bifosfonatos. En pacientes sin compromiso visceral se recomienda (categoría 1) alguna de las siguientes opciones: enzalutamida, abiraterona con prednisona, docetaxel con prednisona, radium-223 (en pacientes con metástasis óseas sintomáticas), terapia hormonal secundaria (antiandrógenos, suspensión de antiandrógenos, ketokonazol o corticoides). En compromiso visceral se recomienda (categoría 1) alguna de las siguientes alternativas: docetaxel con prednisona, enzalutamida, abiraterona con prednisona, mitoxantrone o involucrar al paciente en un ensayo clínico (1). Cuando el dolor óseo no es controlado con el tratamiento sistémico se puede usar la radioterapia a las metástasis óseas (2).

\section{Referencias}

1. National Comprehensive Cancer Network (NCCN). NCCN Clinical practice guidelines in oncology. http://www.ncen.org/ professionals/physician_gls/f_guidelines.asp (Acceso en junio 14, 2015)

2. Howlader N, Noone AM, Krapcho M, Neyman N, Aminou R, Altekruse SF, et al. SEER Cancer Statistics Review, 1975 2009 (Vintage 2009 Populations), National Cancer Institute. Bethesda, MD,http://seer.cancer.gov/csr/1975_2009_pops09/, based on November 2011 SEER data submission, posted to the SEER web site, 2012.

3. Humphrey PA. Cancers of themalereproductiveorgans. In: World Cancer Report, Stewart BW, Wild CP (Eds), World Health Organization, Lyon 2014.

4. Villegas CR, Chacon JA, Sánchez T. Sobrevida en cáncer de próstata de una población del centro de Colombia. Acta Med Colomb 2015; 40: 101-108.

5. Rebelo MS RP, Souza MC et al. Sobrevida geral em cinco anos de pacientes com câncer de próstata, assistidos no Instituto Nacional de Câncer / Hospital do Câncer I, no Rio de Janeiro, para o período de 1990 a 1994. In: Instituto Nacional de Câncer Ministério de Saúde; 2002. 